\title{
The differential outcomes procedure can overcome self-bias in perceptual matching
}

\author{
Luis J. Fuentes ${ }^{1} \cdot$ Jie Sui $^{2}$ - Angeles F. Estévez ${ }^{3}$ Glyn W. Humphreys ${ }^{2}$
}

Published online: 10 July 2015

(C) Psychonomic Society, Inc. 2015

\begin{abstract}
There are biases in perceptual matching between shapes and labels referring to familiar others, compared with when the labels refer to unfamiliar people. We assessed whether these biases could be affected by differential feedback (using the differential outcomes procedure [DOP]) compared with when feedback is provided using a nondifferential outcomes procedure (NOP). Participants formed associations between simple geometric shapes and labels referring to people the participant did or did not know (self, best friend, other). Subsequently, the task was to match a label to one of two shapes shown on a trial. When feedback for correct responses was given following the NOP condition, matches were faster to known people (self and friend) compared with those to an unknown person (stranger). However, this advantage for known personal relations was eliminated when participants were given feedback for correct responses following the DOP condition. The data are consistent with prior work showing that the DOP can facilitate the learning of taxing associations (for the stranger stimuli relative to the familiar self and friend stimuli). In addition, the results suggest that the facilitated perceptual matching for stimuli associated to individuals known personally may reflect better individuation of the
\end{abstract}

Luis J. Fuentes

lfuentes@um.es

Glyn W. Humphreys

glyn.humphreys@psy.ox.ac.uk

1 Department of Psychology, and Regional Campus of International Excellence "Campus Mare Nostrum", University of Murcia, Murcia 30100, Spain

2 Department of Experimental Psychology, University of Oxford, Oxford OX1 3UD, UK

3 Department of Psychology, University of Almería, Almería, Spain association between the shape stimulus and the label, a process enhanced by using a DOP for associations with unfamiliar people.

Keywords Self-bias · Differential outcomes procedure · Associative learning

There is considerable evidence that both memory and perception are strongly modulated by biases towards personally familiar people. For example, there is preferential processing of stimuli relating to ourselves and to familiar others relative to those relating to strangers. In studies of memory, individuals remember both objects and trait terms better if they judge them in relation to themselves compared with other people (Conway, Collins, Gathercole, \& Anderson, 1996). The magnitude of the self-advantage varies according to how close to the self the comparison category is. Overall, there is typically an advantage for the self over close familiar others (Symons \& Johnson, 1997), but this has not always been found (Bowers \& Gilligan, 1979; Keenan \& Ballet, 1980; Seger, Stone, \& Keenan, 2004). In studies of face perception, people are faster and more accurate at making judgements about their own face compared with other faces (Sui, Liu, \& Han, 2009; Sui, Zhu, $\&$ Han, 2006), and responses to faces of friends also benefit relative to those of unfamiliar individuals.

One of the problems with studies using high-level judgements is that it is difficult to tie-down exactly which processes are modulated by self bias. To overcome this problem, we (Sui, He, \& Humphreys, 2012) recently developed a simple associative learning procedure, which is sensitive to effects of the self on perceptual processing. In this paradigm participants are asked to form an association between a shape and a label referring to themselves, a friend, or a stranger (e.g., circle-you; square-friend; triangle-stranger). After just a few learning 
trials, a perceptual matching task is performed in which participants have to judge whether a shape-label pair was one of the original set (circle-you) or if it is a new pairing (circlestranger). Perceptual matching is substantially more efficient for stimuli associated to the familiar people (the self but also familiar others) compared with stimuli associated to unfamiliar people (Sui et al., 2012). Furthermore, this advantage for self-related stimuli interacts with perceptual degradation of the associated shape: reducing the contrast of the shape disrupts the matching of shape-label pairs associated with other people more than the matching of self-related stimuli. This last result is consistent with self-association enhancing the perceptual processing of shapes, so that the shapes require less contrast to be matched.

Differences in the ease of perceptual matching for stimuli related to the self and familiar others relative to unfamiliar others are supported by evidence on brain imaging where regions linked to attentional control are more activated for unfamiliar others. Sui, Rotshtein, and Humphreys (2013) used fMRI to measure brain activity in the matching task. Responses to items linked to familiar others (e.g., the self) increased activation in the ventromedial prefrontal cortex (vmPFC) and the left posterior superior temporal sulcus (LpSTS) compared with when the stimuli linked to unfamiliar others. In contrast, responding to stimuli relating to unfamiliar others was associated with increased activity in a frontoparietal "attentional control" network (Corbetta \& Shulman, 2002). This last result is consistent with more attentional effort being required to judge the task associations (shape and label) for other people. In the present study, we examined whether these more difficult association effects are modulated by how learning is induced.

Work on animal learning has pointed to the importance of several factors that determine the ease of forming new associations. One critical factor is based on whether the learning is linked to a "differential outcome." Differential outcome has been studied in conditional discriminative learning tasks in which a correct choice response to a specific stimulusstimulus association is reinforced with a particular reinforcer or "outcome" (Trapold, 1970; Trapold \& Overmier, 1972). Typically such tasks involve the presentation of a stimulus, the "sample" stimulus, which must be associated with another stimulus, usually referred to as the "comparison" stimulus. Each particular sample-comparison stimulus association requires a specific response that has to be learned. Correct responses are usually reinforced to foster discriminative learning of such specific associations. For instance, in one learning condition, Trapold (1970) associated a particular sample stimulus (e.g., a click) with a particular response lever (e.g., the one in the left) and other sample stimulus (e.g., a tone) with other response lever (e.g., the one in the right) in a Skinner box. The rats had to learn to press the correct lever depending on the sample stimulus that was presented on each trial.
Correct responses were reinforced with a particular outcome (e.g., pellets). That standard discriminative learning procedure was compared with the differential outcomes procedure (DOP). In the DOP, the design was similar to the one previously described except that each particular sample stimulus/ correct choice response was followed by a distinct and unique outcome. Across trials, the rats learned that, in the presence of a click, a press of the left lever systematically led to one type of outcome (e.g., pellets), whereas in the presence of a tone, a press of the right lever systematically led to a different outcome (e.g., sucrose). The DOP had two important effects on learning: 1) the rate of acquiring the association accelerated, and 2) the final accuracy level was greater under the DOP than under the standard learning procedure.

Since then, the DOP has been used to enhance both conditional discriminative learning and memory, in both animals and humans (for reviews, see Urcuoli, 2005; López-Crespo \& Estévez, 2013; Savage, 2001). In humans, the bulk of studies demonstrate that typically developing children and young adults benefit from DOP in conditional discriminative learning tasks, when performance is compared with a nondifferential outcome procedure (hereafter NOP), a control condition more appropriate than the standard learning procedure used in Trapold's original study. In the NOP, the control condition used here, the outcomes (reinforcers) are the same as those in the DOP, but they are provided in a random way (Estévez \& Fuentes, 2003; Estévez, Fuentes, Mari-Beffa, González, \& Álvarez, 2001; Estévez et al., 2007; Maki, Overmier, Delos, \& Gutmann, 1995; Mok \& Overmier, 2007; Mok, Thomas, Lungu, \& Overmier, 2009). The benefits of using the DOP also have been observed in people with discriminative learning difficulties, such as those with Down's syndrome (Estévez, Overmier, Fuentes, \& González, 2003), adults with Prader-Willi syndrome (Joseph, Overmier, \& Thompson, 1997), or children born prematurely (Martínez et al., 2012). Further studies have demonstrated that the DOP is effective at improving memory-based performance in healthy children (Martínez, Estévez, Fuentes, \& Overmier, 2009; Martínez, Flores, González-Salinas, Fuentes, \& Estévez, 2013), young adults (Martella, Plaza, Estevez, Castillo, \& Fuentes, 2012; Plaza, Estévez, LópezCrespo, \& Fuentes, 2011), and older adults (López-Crespo, Plaza, Fuentes, \& Estévez, 2009), as well as individuals with memory-based deficits, such as Korsakoff's syndrome (Hochhalter, Sweeney, Bakke, Holub, \& Overmier, 2000) or Alzheimer's disease (Plaza, López-Crespo, Antúnez, Fuentes, \& Estévez, 2012). It is of some interest to note that selfassociation has been shown to improve memory in patients with severe amnesia (Sui \& Humphreys, 2013).

The DOP benefits have been explained at both the cognitive and neural levels (for reviews, see Savage, 2001; Savage $\&$ Ramos, 2009). The consistent pairing of a sample stimulus with a unique outcome, which is characteristic of the 
differential outcomes procedure, results in the activation of specific conditioned reinforcement expectations for each sample stimulus. That is, when a sample stimulus is presented an expectation of the unique outcome associated with that sample stimulus is activated. That expectation can be conceptualized as a prospective memory "representation," elicited by the sample stimulus, of which outcome will be forthcoming (Savage, 2001). These expectancies provide an additional source of information as learning takes place and as the expectancies are robust to delays and/or working memory demands, correct responding is facilitated. Ramirez and Savage (2007) showed that the basolateral amygdala and the orbitofrontal cortex are critical for the development and maintenance, respectively, of outcome expectancies in animals when hedonic (e.g., food) reinforcers are used. In contrast, under more standard nondifferential outcomes conditions, there is only one source of information that can guide correct responding. Under these conditions, participants must rely on their retrospective memory for the previously presented sample stimulus to solve the task successfully (Savage, 2001). The hippocampus has been mainly involved in this process in both animal (Savage, Buzzetti, \& Ramirez, 2004) and human (Mok et al., 2009) studies.

Interestingly, the perceptual matching procedure developed by Sui and colleagues (Sui et al., 2012) has the structure of a conditional discriminative learning task. Participants have to learn that a particular label (the sample stimulus) is linked to a particular shape (the comparison stimulus) and that association (represented in the target display) requires a specific correct choice response (to press a particular key). Explicit feedback after a correct response may be provided (e.g., the word CORRECT, the reaction time), or when feedback is not made explicit, the participants notice when their responses are correct and that knowledge can operate as an implicit feedback stimulus.

In this procedure, as in standard animal learning procedures, correct responses are usually reinforced. However, for the self and familiar others there may be a more differentiated linkage formed between the sample and comparison stimuli than is for the case for stimuli related to unfamiliar people, given that we carry more information about known than unknown people and that we may have more motivational interest in familiar individuals. This advantage may be reduced if differential outcomes learning can be introduced in the Sui et al. matching procedure, and this may improve performance mainly for unfamiliar people. This was the purpose of this study, where we introduced the DOP into the personal association task of Sui et al. (2012). We also examined the effects of the self- and other-associations under conditions in which participants had to select the stimulus matching a label (rather than having participants decide whether a single shape and label matched). Each display contained a label and two shapes, and participants selected the shape matching the label.

The effects of the DOP procedure have been found particularly when the task is relatively demanding (Estévez et al.,
2001, 2007; Legge \& Spetch, 2009; Plaza et al., 2011) and might be more likely to emerge under conditions of competition between the shapes. In the nondifferential outcomes version (the NOP condition) participants received outcomes after correct self- and other-person matches in a random way. In the DOP procedure, participants received individuated outcomes for each sample-comparison correct match. We asked whether the DOP procedure selectively benefitted associations to unfamiliar people, consistent with matches to unfamiliar people both being more difficult and attentionally demanding (Sui et al., 2013) and normally less differentiated relative to associations to known individuals.

\section{Method}

\section{Participants}

Thirty-eight undergraduates from the University of Almería (Spain), ranging in age from 18 to 29 years, participated in the experiment. They received course credits for their participation. All were naïve with respect to the purposes of the experiment and had normal or corrected-to-normal vision.

\section{Stimuli and materials}

The stimuli consisted of three labels (YOU, FRIEND, STRA NGER, in Spanish), which were associated to three target/ distracter shapes (triangle, square, pentagon). For half of the trials, each label was presented with both an associated target shape (e.g., YOU with triangle, FRIEND with square, STRA NGER with pentagon) and a neutral distracter (the circle), i.e., the neutral distracter condition. For the other half of the trials, the distracter shape was one of the stimuli associated with the other two labels (e.g., if YOU was associated with triangle, half of these trials contained a distracter square, associated with FRIEND, and half contained a distracter pentagon, associated with STRANGER), i.e., the incompatible distracter condition. Primary and secondary reinforcers served as outcomes. Primary reinforcers were a pen drive, a massage apparatus, and a key ring. Three photographs of these prizes were used as secondary reinforcers or outcomes.

The stimuli were presented in black on a white background on a colour monitor (VGA) of a PC. The labels (YOU, FRIE ND, STRANGER) were displayed in capital letters in font Courier New 30. The shapes were 3.6-cm wide and 3.6-cm high. Pictures of the outcomes measured approximately $10 \times$ $13 \mathrm{~cm}$ and were presented individually at the centre of the screen along with the text: "You may win a (one of the primary reinforcers)!" which appeared above the picture after a correct response. E-prime (Schneider, Eschman, \& Zuccolotto, 2002) controlled the presentation of the stimuli as well as the collection of the reaction time (RT) and accuracy data. 


\section{Procedure}

The experiment consisted of two phases. In the association phase, participants were told that the label referring to themselves (YOU) would be associated with a particular shape, e.g., a triangle; the label referring to their named best friend (FRIEND; participants were told to say the name of that person aloud) would be associated with another shape, e.g., a square; and the label referring to an unknown person (STRA NGER) would be associated with a third shape, e.g., a pentagon. Each label and its corresponding shape were presented four times on the screen to allow participants to form the associations. Label and shape links were counterbalanced across participants.

In the experimental phase, participants performed a target localization task. On each trial, a fixation point (a plus sign) was presented in the centre of the screen for $500 \mathrm{~ms}$. The fixation display was followed by the target display for $100 \mathrm{~ms}$, which was then followed by a blank screen for $1200 \mathrm{~ms}$ or until the participant responded, whichever occurred first. The target display contained the label on the upper part of the screen and two shapes, the target and the distracter shape, in the lower part of the screen (see Fig. 1 for an example of an experimental trial). The target was always the shape associated with the current label for a particular participant. For half the trials the distracter shape was the one that was not associated to any label (the neutral distracter condition) and for the other half it was one of the shapes associated with another labels (the incompatible distracter condition). The target shape was located on the right side of the screen for half the trials and on the left side for the other half. Participants responded by pressing the key " $\mathrm{M}$ " if the target was on the right side and the key " $\mathrm{C}$ " if the target was on the left side. A picture of a prize (the outcome) always followed a correct response for $500 \mathrm{~ms}$, whereas a blank screen followed incorrect responses for the same duration. Participants were told that the prizes would be accumulated with correct responses and raffled off at the end of the experiment as a present for participation. Previous studies have demonstrated that raffling off the prizes at the end of the study is an effective way of promoting correct responses and therefore assessing the effects of the DOP (López-Crespo et al., 2009; Plaza et al., 2011). Fast responses were encouraged as participants were only allowed to press the response key within the aforementioned time frame. A blank screen lasting $200 \mathrm{~ms}$ followed the reinforcer or the time-out period and then the next trial started.

Half of the participants were randomly assigned to the differential outcomes procedure (DOP) condition and half to the nondifferential outcomes procedure (NOP) condition. In the DOP condition, each label/shape combination was followed by a specific and unique outcome throughout the experiment. In the NOP condition, the same outcomes used in the DOP were used for each label/shape combination, but now they were provided in a random manner. All participants performed 6 blocks of 72 experimental trials. There were 216 trials with the neutral distracter and 216 with an incompatible distracter, 36 trials in each possible combination: friend-distracter with label "you"; stranger-distracter with label "you"; youdistracter with label "friend"; stranger-distracter with label "friend"; you-distracter with label "stranger"; and frienddistracter with label "stranger." The instructions highlighted both speed and accuracy in responding.

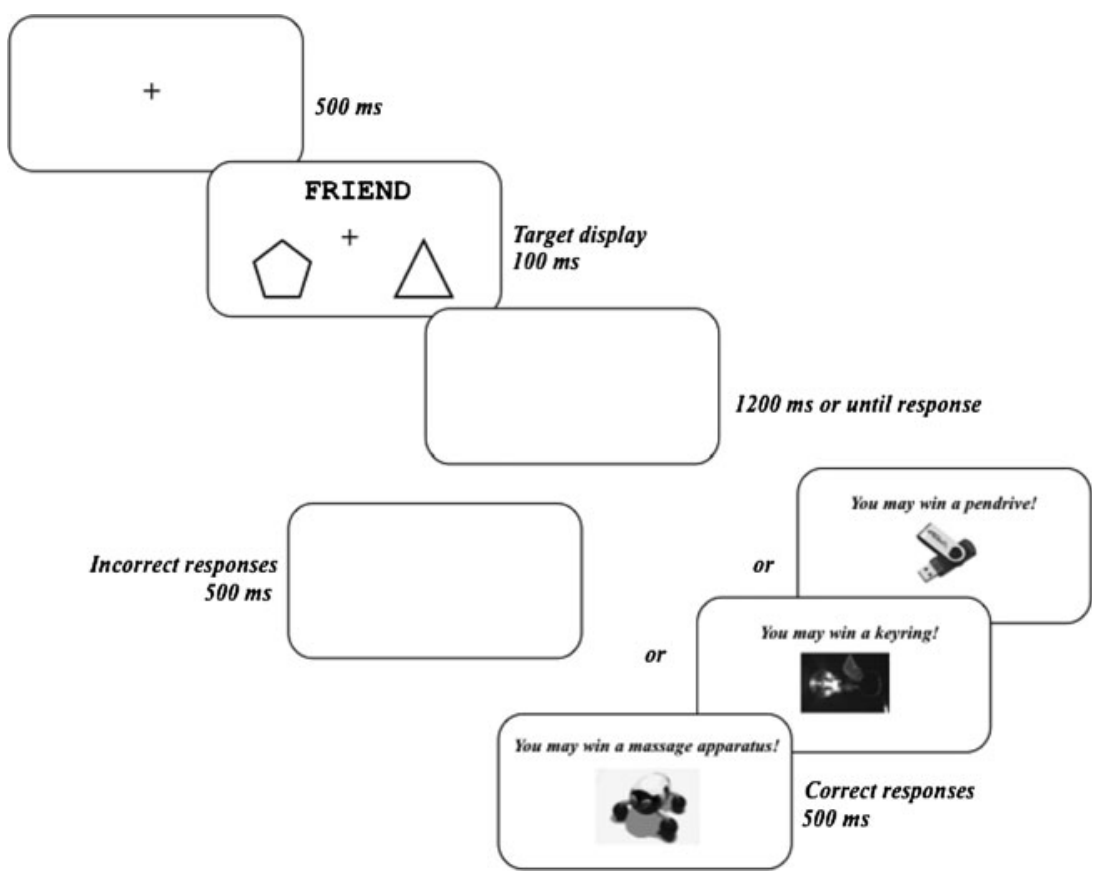

Fig. 1 Sequence of stimuli and exposition durations in the experiment 


\section{Results}

Table 1 shows the percentage of correct responses and the mean of the correct RTs. Data from both dependent variables were submitted to a $2 \times 3 \times 2$ mixed ANOVA with Outcome Procedure type (DOP, NOP) as the between-participants factor, and Label (you, friend, stranger) and Distracter type (incompatible, neutral) as within-participants factors. When necessary, post hoc comparisons were calculated by the Least Significant Difference (LSD) test. The significance level to reject the null hypothesis was set at $p \leq 0.05$. GreenhouseGeisser $(\mathrm{G}-\mathrm{G})$ corrections were applied to the degrees of freedom of the repeated factor in order to correct for any violation of the sphericity assumption, as assessed by the Mauchly's W test.

\section{Accuracy analysis}

The analysis conducted on response accuracy showed a significant main effect of Distracter type $[F(1,36)=207.20, p<$ $\left.0.001, \eta^{2} \mathrm{p}=0.852\right]$. Participants were less accurate in the incompatible distracter condition (77\% correct) than in the neutral distracter condition (93\% correct). The Distracter type $\times$ Outcome Procedure type interaction also was significant $\left[F(1,36)=7.33, p<0.05, \eta^{2} \mathrm{p}=0.169\right]$. The interaction analysis indicated that the interference effect (incompatible minus neutral) was reduced in the DOP condition (interference effect $=13$ ) compared with the NOP (interference effect $=19)\left[F(1,36)=7.32, p<0.05, \eta^{2} p=0.169\right]$. The

Table 1 Mean percentages of correct responses, standard error of the mean (SE), and mean correct RTs (in milliseconds) as a function of Outcome Procedure type (DOP, NOP), Label (you, friend, stranger), and Distracter type (incompatible, neutral)

\begin{tabular}{|c|c|c|c|c|c|c|}
\hline & \multicolumn{2}{|c|}{ YOU } & \multicolumn{2}{|c|}{ FRIEND } & \multicolumn{2}{|c|}{ STRANGER } \\
\hline & M & SE & M & SE & M & SE \\
\hline \multicolumn{7}{|l|}{ Accuracy } \\
\hline \multicolumn{7}{|c|}{ Differential (DOP) } \\
\hline Incompatible & 80 & 3.0 & 78 & 3.1 & 79 & 3.3 \\
\hline Neutral & 92 & 2.8 & 91 & 2.1 & 92 & 2.4 \\
\hline \multicolumn{7}{|c|}{ Nondifferential (NOP) } \\
\hline Incompatible & 78 & 3.1 & 78 & 2.5 & 70 & 2.7 \\
\hline Neutral & 95 & 0.6 & 95 & 1.0 & 92 & 1.0 \\
\hline \multicolumn{7}{|l|}{ Reaction times } \\
\hline \multicolumn{7}{|c|}{ Differential (DOP) } \\
\hline Incompatible & 652 & 27.0 & 660 & 15.9 & 662 & 21.8 \\
\hline Neutral & 596 & 20.1 & 611 & 13.2 & 624 & 17.1 \\
\hline \multicolumn{7}{|c|}{ Nondifferential (NOP) } \\
\hline Incompatible & 661 & 19.1 & 663 & 24.3 & 728 & 23.9 \\
\hline Neutral & 611 & 17.0 & 615 & 18.3 & 646 & 19.0 \\
\hline
\end{tabular}

trend was for this effect to be strongest for stranger-associated stimuli, but no other effects were statistically significant.

\section{Reaction time analysis}

The analysis conducted on the RT data showed significant main effects of Label $\left[F(1.5,55.1)=6.81, p<0.01, \eta^{2} \mathrm{p}=\right.$ $0.159]$ and Distracter type $\left[F(1,36)=98.38, p<0.001, \eta^{2} \mathrm{p}=\right.$ $0.732]$. Regarding the label effect, post hoc comparisons revealed that the label "stranger" (665 ms) produced longer RTs than the labels "you" (630 ms) and "friend" (637 ms) ( $p \mathrm{~s}<$ $0.05)$, but the latter two conditions did not differ significantly $(p>0.05)$. Regarding the distracter type effect, the incompatible distracter produced longer RTs than the neutral distracter (671 vs. $617 \mathrm{~ms}$ ). The main effect of Outcome Procedure type was not significant $(F<1)$. However, there was a significant Outcome Procedure type $\times$ Label $\times$ Distracter type interaction $\left[F(2,72)=5.10, p<0.01, \eta^{2} \mathrm{p}=0.124\right]$. To further analyze the three-way interaction, we first looked at the Outcome Procedure type $\times$ Label interaction for each type of distracter. With the neutral distracter, the Outcome Procedure type $\times$ Label interaction was not significant $(F<1)$. Only the main effect of Label was significant $[F(1.5,54.5)=9.46, p<0.01$, $\left.\eta^{2} \mathrm{p}=0.208\right]$; that is, RTs to the label "stranger" were longer than RTs to both the labels "you" and "friend." That pattern of results changed with the incompatible distracter. Although the main effect of Label was significant $[F(1.6,58.6)=4.73, p<$ $\left.0.05, \eta^{2} \mathrm{p}=0.116\right]$, this effect was now modulated by a significant Outcome Procedure type $\times$ Label partial interaction $\left[\mathrm{F}(2,72)=3.32, p<0.05, \eta^{2} \mathrm{p}=0.084\right]$. The partial interaction analysis revealed a significant Label effect only in the NOP condition $\left[F(2,36)=6.04, p<0.01, \eta^{2} \mathrm{p}=0.251\right]$. That is, there were longer RTs with the label "stranger" compared with the labels "you" and "friend" ( $p$ s $<0.05)$. However, when differential outcomes were arranged (in the DOP condition) significant differences between the labels were no longer observed ( $p s>0.05)$. That is, RTs with the label "stranger" were now reduced to the level of those observed with the labels "you" and "friend."

A second analysis of the three-way interaction took the difference between the incompatible and the neutral distracter, i.e., the interference effect, as the dependent variable. The Outcome Procedure type $\times$ Label interaction was again significant $\left[F(2,72)=5.10, p<0.01, \eta^{2} p=0.124\right]$ (Fig. 2). The interference effect with the label "stranger" was greater than the interference effect with the labels "you" and "friend" ( $p s<$ $0.05)$ in the NOP condition $\left[F(2,36)=3.76, p<0.05, \eta^{2} \mathrm{p}=\right.$ $0.173]$. In contrast, the interference effect did not differ significantly between the three labels in the DOP condition ( $p s>$ $0.05)$. In addition, only the interference effect with the label "stranger" showed significant differences between the conditions DOP and NOP $\left[F(1,36)=8.72, p<0.01, \eta^{2} \mathrm{p}=0.195\right]$; 


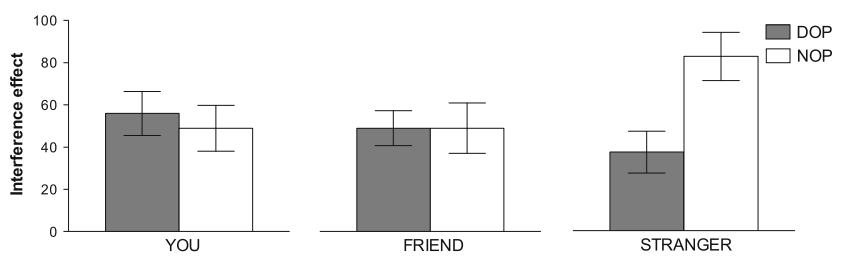

Fig. 2 Interference effects (incompatible minus neutral) as a function of Outcome Procedure type (DOP, NOP) and Label (you, friend, stranger). Error bars represent the standard error of the mean. DOP $=$ differential outcomes procedure. $\mathrm{NOP}=$ nondifferential outcomes procedure

the interference effect was greater under the NOP than under the DOP condition.

To further analyze the modulation of Outcome Procedure type on the interference effect observed with the label "stranger," we separated interference coming from the incompatible distracter associated with the label "you" (ID_YOU) from that coming from incompatible distracter associated with the label "friend" (ID_FRIEND). Only the main effect of Outcome Procedure type was significant $[F(1,36)=8.72, p$ $\left.<0.01, \eta^{2} \mathrm{p}=0.195\right]$, indicating that the DOP condition improved performance (reducing the interference effect) to a similar extent for both ID_YOU and ID_FRIEND trials (Fig. 3).

\section{Discussion}

Participants formed associations between a personal label and a shape. Subsequently, they had to select which of two shapes matched a label. When participants were given the same set of rewards randomly across each match condition, there were advantages in selecting shapes associated with familiar others (the self, the participant's best friend) compared with an unfamiliar other. This replicates prior studies where single shapes have to be matched with an associated label (Sui et al., 2012). The novel result is that the cost for unfamiliar others was eliminated when participants were given differential outcomes feedback in the selection task. Under the DOP condition,
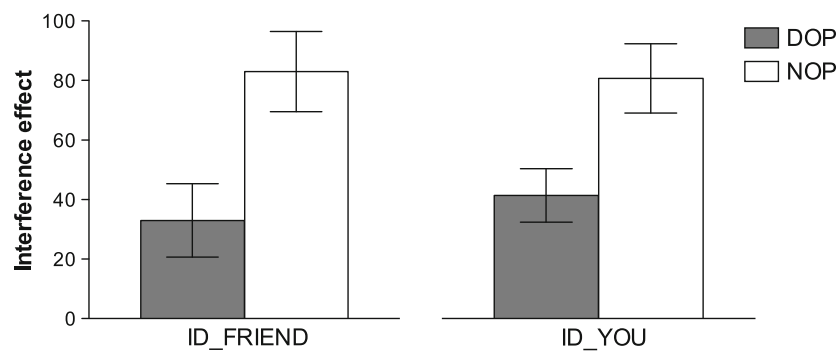

Fig. 3 Interference effects (incompatible minus neutral) produced by ID_YOU and ID_FRIEND distracter stimuli paired with the label "stranger" for conditions DOP and NOP. Error bars represent the standard error of the mean. ID YOU $=$ incompatible distracter associated with the label "you." ID_FRIEND = incompatible distracter associated with the label "friend." DOP $=$ differential outcomes procedure. $\mathrm{NOP}=$ nondifferential outcomes procedure participants received individuated feedback (a different outcome was given as feedback for each association). Studies with both animal and humans have shown that associative learning is facilitated under DOP conditions (López-Crespo \& Estévez, 2013), particularly under conditions where learning is more taxing and attentionally demanding (Estévez et al., 2001, 2007; Plaza et al., 2011).

Work using fMRI has provided converging evidence that the task of matching shape-label associations for unfamiliar people is more attentionally demanding than matching similar associations with familiar people (particularly relative to selfmatches; Sui et al., 2013). The present data fit with this, because the DOP was selectively beneficial for matching and selecting targets associated with unfamiliar relative to familiar others. We suggest that having differential outcomes feedback helps to form stronger associative links between sample and comparison stimuli, and, under the conditions examined here, this benefits the ability to select the target shape linked to the label for the unfamiliar person. The converse of this result is that the links formed to familiar others (self, best friend) take a form of differential learning from the outset, even under conditions of non-differential outcomes. We suggest that this reflects the ability of participants to form individuated tokens for familiar people, which can be associatively linked with the shapes. This might in turn reflect the greater knowledge we have about familiar others or the greater motivational significance of familiar compared with unfamiliar people. A question for future research will be to evaluate which are the critical factors that benefit associative learning and matching for familiar relative to unfamiliar others.

One other point to note is, while studies using matching of single shapes with labels have demonstrated benefits for selfrelated stimuli over stimuli associated with familiar others (best friend, mother; see Sui et al., 2012), we failed to find reliable differences. We propose that this reflects a difference in the procedures employed compared with those used previously. Participants have to use the association to select between two stimuli, essentially making the current task one of cued retrieval. Provided that there was a sufficient difference in association strength between (1) the shapes linked to familiar target labels (self, friend) and (2) the strength of the association of the same label to the critical related distracter (respectively friend for the self target, or self for the friend target), then the cue to select a target based on differential familiarity may be equated. For example, the self label may be strongly related to its own shape, but also strongly differentiated from the friend shape. This may mean that there is relatively low competition for a self target from a friend distracter, but also low competition for a friend target from a self distracter. In contrast, if matches between single shapes and labels reflect the absolute strength of that association, and not the relative difference between one association and that to other stimuli, then self-biases may emerge in experiments using single shape-label matches (if the absolute 
strength of the association is increased for self-related items; Sui et al., 2012). In addition, if the strength of association from the label to the shape is generally weaker for unfamiliar items under NOP learning, then the time to select the unfamiliar item may suffer overall compared with both friend and self stimuli. Moreover, there will be a greater benefit for unfamiliar associations from using DOP learning, as we observed.

It has been found that the effects of self-bias in perceptual matching to associated shapes extends to members of the same in-group (Moradi, Sui, Hewstone, \& Humphreys, 2015), consistent with members of the in-group being assimilated into a representation of the self (Aron, Aron, Tudor, \& Nelson, 1991). In the current experiments it may be that, in addition to the procedure being sensitive to relative differences in familiarity, the participants may have selected closer friends than in prior experiments, as this factor was not controlled, and closer friends may be linked to the individual's self-representation. It is interesting to note that self-biases may vary across different cultures. For example, self-biases in face perception relative to familiar others tend to be larger in Western than Eastern cultures (Sui et al., 2009), likely reflecting the tendencies towards more individualistic vs. more collectivist cognition and with the representations of a friend being more strongly linked to the self in Eastern societies. Following from the current research, we may ask whether some of these cultural differences themselves reflect differential reward histories for information about the self vs. other people. Is it the case that Western societies tend to use more differential reward systems for information linked to the self whilst group-level rewards are given in Eastern cultures, reducing differences between the self and close others? Clearly, these are questions for future research.

Finally, we note that DOP procedures have been used successfully to remediate a variety of learning problems, including those affecting patients with Korsakoff's syndrome (Hochhalter et al., 2000) and Alzheimer's disease (Plaza et al., 2012). Similarly, self-reference effects have been shown to improve the memory of amnesic individuals (Sui \& Humphreys, 2013). It would be interesting and potentially important to assess the effects of combining self-reference and DOP to stimuli to examine how these two effects combine and whether there may be interactive gains from coupling DOP and the self.

We conclude that linkages with familiar others can benefit the formation of shape-label associations, and the use of these labels in selection tasks, due to the associations being strongly individuated (for the self and a best friend). For unfamiliar others, there is a cost of having reduced individuated during learning, but this can be overcome by introducing differential outcomes procedures. The results highlight the role of individuated associations for enhancing learning and subsequent selection performance.
Acknowledgments This work was supported by grants from the Economic and Social Research Council to JS and GWH, from the European Research Council to GWH, from the National Science Foundation China to JS, and from the Spanish Ministry of Economy and Competitivity to AFE (PSI2012-39228) and LJF (PSI2014-53427-P) and Leverhulme Trust to LJF.

\section{References}

Aron, A., Aron, E. N., Tudor, M., \& Nelson, G. (1991). Close relationships as including other in self. Journal of Personality and Social Psychology, 60(2), 241-253.

Bowers, G. H., \& Gilligan, S. G. (1979). Remembering information related to one's self. Journal of Research in Personality, 13, 420-432.

Conway, M. A., Collins, A. F., Gathercole, S. E., \& Anderson, S. J. (1996). Recollections of true and false autobiographical memories. Journal of Experimental Psychology: General, 125, 69-95.

Corbetta, M., \& Shulman, G. L. (2002). Control of goal directed and stimulus-driven attention in the brain. Nature Reviews Neuroscience, 3, 201-215.

Estévez, A. F., \& Fuentes, L. J. (2003). Differential outcomes effect in four-year- old children. Psicológica, 24, 159-167.

Estévez, A. F., Fuentes, L. J., Mari-Beffa, P., González, C., \& Álvarez, D. (2001). The differential outcomes effect as useful tool to improve conditional discrimination learning in children. Learning and Motivation, 1(32), 48-64. doi:10.1006/lmot.2000.1060

Estévez, A. F., Overmier, J. B., Fuentes, L. J., \& González, C. (2003). Differential outcomes effect in children and adults with Down syndrome. American Journal on Mental Retardation, 108, 108-116. doi:10.1352/0895-8017(2003)108<0108

Estévez, A. F., Vivas, A. B., Alonso, D., Marí-Beffa, P., Fuentes, L. J., \& Overmier, J. B. (2007). Enhancing challenged students' recognition of mathematical relations through differential outcomes training. The Quarterly Journal of Experimental Psychology, 60(4), 571580. doi:10.1080/17470210600820039

Hochhalter, A. K., Sweeney, W. A., Bakke, B. L., Holub, R. J., \& Overmier, J. B. (2000). Improving face recognition in alcohol dementia. Clinical Gerontologist, 22(2), 3-18. doi:10.1300/ J018v22n02_02

Joseph, B., Overmier, J. B., \& Thompson, T. (1997). Food and nonfood related differential outcomes in equivalence learning by adults with Prader-Willi syndrome. American Journal on Mental Retardation, 101, 374-386.

Keenan, J. M., \& Ballet, S. D. (1980). Memory for personally and socially relevant significant events. In R. S. Nickerson (Ed.), Attention and performance 8. Hissdale, N.J: Lawrence Erlbaum Assoc.

Legge, E. L. G., \& Spetch, M. L. (2009). The differential outcomes effect (DOE) in spatial localization: An investigation with adults. Learning and Motivation, 40, 313-328. doi:10.1016/j.lmot.2009.03.002

López-Crespo, G., \& Estévez, A. F. (2013). Working memory improvement by the differential outcomes procedure. In S. H. ClairThompson (Ed.), Working memory: Developmental differences, component processes, and improvement mechanisms (pp. 145157). New York: Nova Publishers.

López-Crespo, G., Plaza, V., Fuentes, L. J., \& Estévez, A. F. (2009). Improvement of age-related memory deficits by differential outcomes. International Psychogeriatrics, 21(3), 503-510. doi:10. 1017/S1041610209008576

Maki, P., Overmier, J. B., Delos, S., \& Gutmann, A. J. (1995). Expectancies as factors influencing conditional discrimination performance of children. The Psychological Record, 45, 45-71.

Martella, D., Plaza, V., Estevez, A. F., Castillo, A., \& Fuentes, L. J. (2012). Minimizing sleep deprivation effects in healthy adults by 
differential outcomes. Acta Psychologica, 139, 391-396. doi:10. 1016/j.actpsy.2011.12.013

Martínez, L., Estévez, A. F., Fuentes, L. J., \& Overmier, J. B. (2009). Improving conditional discrimination learning and memory in fieyear-old children: DOE using different types of reinforcement. The Quarterly Journal of Experimental Psychology, 62, 1617-1630. doi: $10.1080 / 17470210802557827$

Martínez, L., Flores, P., González-Salinas, C., Fuentes, L. J., \& Estévez, A. F. (2013). The effects of differential outcomes and different types of consequential stimuli on seven-year-old children's discriminative learning and memory. Learning \& Behavior, 41, 298-308. doi:10. 3758/s13420-013-0105-y

Martínez, L., Marí-Beffa, P., Roldán-Tapia, D., Ramos-Lizana, J., Fuentes, L. J., \& Estévez, A. F. (2012). Training with differential outcomes enhances discriminative learning and visuospatial recognition memory in children born prematurely. Research in Developmental Disabilities, 33, 76-84. doi:10.1016/j.ridd.2011.08. 022

Mok, L. W., \& Overmier, J. B. (2007). The differential outcomes effect in normal human adults using a concurrent-task within-subjects design and sensory outcomes. The Psychological Record, 57, 187-200.

Mok, L. W., Thomas, K. M., Lungu, O. V., \& Overmier, J. B. (2009). Neural correlates of cue-unique outcome expectations under differential outcomes training: An fMRI study. Brain Research, 1265, 111-127. doi:10.1016/j.brainres.2008.12.072

Moradi, Z., Sui, J., Hewstone, M., \& Humphreys, G. W. (2015). In-group modulation of perceptual matching. Psychonomic Bulletin and Review. doi:10.3758/s13423-014-0798-8

Plaza, V., Estévez, A. F., López-Crespo, G., \& Fuentes, L. J. (2011). Enhancing recognition memory in adults through differential outcomes. Acta Psychologica, 136, 129-136. doi:10.1016/j.actpsy. 2010.11.001

Plaza, V., López-Crespo, G., Antúnez, C., Fuentes, L. J., \& Estévez, A. F. (2012). Improving delayed face recognition in Alzheimer's disease by differential outcomes. Neuropsychology, 26(4), 483-489. doi:10. 1037/a0028485

Ramirez, D. R., \& Savage, L. M. (2007). Differential involvement of the basolateral amygdala, orbitofrontal cortex, and nucleus accumbens core in the acquisition and use of reward expectancies. Behavioral Neuroscience, 121, 896-906. doi:10.1037/0735-7044.121.5.896

Savage, L. M. (2001). In search of the neurobiological underpinnings of the differential outcomes effect. Integrative Physiological and Behavioral Science, 36, 182-195. doi:10.1007/BF02734092

Savage, L. M., Buzzetti, R. A., \& Ramirez, D. R. (2004). The effects of hippocampal lesions on learning, memory, and reward expectancies.
Neurobiology of Learning and Memory, 82, 109-119. doi:10.1016/j. nlm.2004.05.002

Savage, L. M., \& Ramos, R. L. (2009). Reward expectation alters learning and memory: The impact of the amygdala on appetitive-driven behaviors. Behavioural Brain Research, 198, 1-12. doi:10.1016/j. bbr.2008.10.028

Schneider, W., Eschman, A., \& Zuccolotto, A. (2002). E-Prime user's guide. Pittsburgh, PA: Psychology Software Tools, Inc.

Seger, C. H., Stone, M., \& Keenan, J. P. (2004). Cortical activation during judgments about the self and another person. Neuropsychologia, 42, $1168-1177$.

Sui, J., \& Humphreys, G. W. (2013). Self-referential processing is distinct from semantic elaboration: Evidence from long-term memory effects in a patient with amnesia and semantic impairments. Neuropsychologia, 51, 2663-2673.

Sui, J., He, X., \& Humphreys, G. W. (2012). Perceptual effects of social salience: Evidence from self-prioritization effects on perceptual matching. Journal of Experimental Psychology: Human Perception and Performance, 38, 1105-1117. doi:10.1037/ a0029792

Sui, J., Liu, C. H., \& Han, S. (2009). Cultural difference in neural mechanisms of self-recognition. Social Neuroscience, 4, 402-411. doi:10. 1080/17470910802674825

Sui, J., Rotshtein, P., \& Humphreys, G. W. (2013). Coupling social attention to the self forms a network for personal significance. Proceedings of the National Academy of Sciences of the United States of America, 110, 7607-7612. doi:10.1073/pnas. 1221862110/-/DCSupplemental

Sui, J., Zhu, Y., \& Han, S. (2006). Self-face recognition in attended and unattended condition: An event-related brain potential study. Neuroreport, 17, 423-427. doi:10.1097/01.wnr.0000203357. 65190.61

Symons, C. S., \& Johnson, B. T. (1997). The self reference effect in memory: A meta-analysis. Psychological Bulletin, 121, 371-394.

Trapold, M. A. (1970). Are expectancies based upon different positive reinforcing events discriminably different? Learning and Motivation, 1, 129-140. doi:10.1016/0023-9690(70)90079-2

Trapold, M. A., \& Overmier, J. B. (1972). The second learning process in instrumental learning. In A. H. Black \& W. F. Prokasy (Eds.), Classical conditioning II: Current theory and research (pp. 427452). New York: Appleton-Century Crofts.

Urcuoli, P. J. (2005). Behavioral and associative effects of differential outcomes in discrimination learning. Learning \& Behavior, 33, 121. doi:10.3758/BF03196047 